\title{
IMMANUEL KANT: EL GIRO COPERNICANO COMO ONTOLOGÍA DE LA EXPERIENCIA
}

\author{
María Jesús VÁZQUEZ LOBEIRAS \\ Universidad de Santiago de Compostela
}

ABSTRACT: Tomando como guta el problema de la experiencia, en el presente trabajo se ofrece un estudio de la evolución del pensamiento de Kant en el periodo comprendido entre la Dissertatio de 1770 y la Critica de la razbn pura (1781-1787). Los datos obtenidos permiten reconstruir las etapas evolutivas del giro copernicano que aqui hemos denominado 'giro de la sensibilidad' (1770) y 'giro del entendimiento' (1772-1781). El análisis proporciona recursos para la interpretación del idealismo trascendental como ontologia de la experiencia y para apreciar algunas de las aportaciones que convierten a Kant en la cima de la modernidad, sobre todo en relación con la constitución de la razón finita.

PALABRAS ClAVES: experiencia, objeto, idealismo trascendental, ontologia, evolución del pensamiento de Kant, razón finita.

1. El problema de la experiencia en el contexto del pensamiento moderno

El pensamiento de Kant fructifica en el núcleo mismo de las tensiones que recorren la primera parte de la modernidad. En el horizonte intelectual de este pensador cabe señalar, al menos, las siguientes líneas de fuerza: la coronación del proyecto de la ciencia moderna con Newton, el esfuerzo de Wolff-Baumgarten en pos del ideal cartesiano y leibniziano de un sistema universal del conocimiento y la demoledora crítica de Hume a los instrumentos conceptuales básicos sobre los que se fundamentan, tanto el proyecto de la ciencia como el de la metafísica modernas.

El objetivo del presente estudio consiste en una aproximación a la génesis del pensamiento de Kant tomando como hilo conductor el problema de la experiencia. La peculiar posición de Kant como cima de la modernidad se debe a su 
capacidad para elaborar una respuesta a estè problema, que constituye, al mismo tiempo, el fundamento de una nueva ontología y la explicación tal vez más compleja y completa que en filosofía se haya dado al problema del conocimiento.

En la medida en que el entendimiento humano haya de ser fuente de algún tipo de certeza que vaya más allá de la mera autoconciencia, debe apuntar a algo que no sea él mismo. En el punto de partida del empirismo, con Locke, ese 'algo' que se sitúa más allá del entendimiento, lo 'otro' en relación con lo cual se puede plantear la cuestión de la certeza como objetividad que rebasa los límites del cogito, recibe el nombre de 'experiencia'. El programa kantiano admitiría ser interpretado como el último eslabón en la cadena de filosofias de la experiencia que comienza con Locke. Desde este punto de vista, la aportación del empirismo habría consistido fundamentalmente en convertir la experiencia en un grave problema y en haber conducido el enigma del conocimiento al callejón sin salida del escepticismo. Conviene, sin embargo, tener presentes las diferencias fundamentales entre el programa empirista y el nuevo programa del idealismo trascendental. En el marco del empirismo, la 'experiencia' se interpreta como el punto de partida del proceso cognoscitivo, mientras que para Kant, en cambio, la experiencia aparece como el resultado, como el producto de la actividad cognoscitiva, en la que necesariamente intervienen como soporte todo el conjunto de condiciones interpuestas por la subjetividad humana. En la Introducción a la Crítica de la razón pura en la versión de la primera edición: «La experiencia es, sin ninguna duda, el primer producto surgido de nuestro entendimiento al elaborar éste la materia bruta de las impresiones sensibles" $\left(C_{r p}, \mathrm{~A} 1\right)^{1}$. Kant mismo, al juzgar su propia posición en relación con el punto de vista de Hume, hace hincapié en la comprensión de la experiencia como un producto, como un punto de llegada y no como un punto de partida. Así, en las páginas que abren paso a la deducción trascendental, en un pasaje añadido en la segunda edición: "[Hume] tampoco advirtió que el mismo entendimiento podría quizá, a través de estos conceptos, ser el autor de la experiencia en la que se hallan sus objetos" (Crp, B 127).

La orientación de la investigación kantiana se hace explícita en la sentencia tantas veces citada del Prólogo a la segunda edición de la Critica de la razón pura:

1 Cito la Critica de la razón pura (=Crp) siguiendo la versión castellana de Pedro Ribas, ed. Alfaguara, Madrid, "1995 ('1978), indicando como es usual entre paréntesis la paginación correspondiente a la primera y segunda edición. 
«Pero, aunque todo nuestro conocimiento empiece con la experiencia, no por eso procede todo él de la experiencia" ( $C r p, \mathrm{~B}$ 1). Empieza, se echa a andar o se abre paso (anbebt) con la experiencia, pero no procede o se origina (entspringt) totalmente en la experiencia. Kant está abordando la posibilidad de una nueva respuesta genética al problema del conocimiento.

El problema del origen del conocimiento habría evolucionado en el desarrollo del empirismo del punto de vista de la causación al punto de vista de la inmediatez. Locke admitía la causación de las ideas por las cosas, Berkeley, siguiendo a Malebranche, habría sustituido la causación material por la causación de la mente divina. Hume habría abandonado definitivamente el argumento de la causación analizando los contenidos elementales de la conciencia desde el punto de vista de su inmediatez ${ }^{2}$. La posición de Kant podría caracterizarse, en términos generales, como el punto de vista de la mediación. Kant establece el principio de que, incluso en el nivel más elemental del conocimiento, en las sensaciones, se da una mediación, la mediación a través de las formas de la sensibilidad o intuiciones puras. El atomismo gnoseológico característico del empirismo, que consideraba las sensaciones aisladas como los primeros datos del conocimiento, se transforma de raíz en la estética trascendental kantiana. En el dato sensible mismo existe elaboración por parte de la conciencia. La experiencia, en su nivel más elemental, aparece capturada, valga la metáfora, por la subjetividad.

\section{Experiencia y razón finita: el giro copernicano}

La noción de experiencia entraña el problema de un tipo de conocimiento adecuado a las capacidades humanas. La atención cada vez mayor al problema de la experiencia acompaña, en el decurso de la modernidad, al paulatino desplazamiento de la razón finita hacia el centro de gravedad de la cuestión epistemológica. En el contexto de la investigación sobre la obra de Kant se ha consagrado la expresión 'giro copernicano' para designar esta transformación del panorama del pensamiento, que traerá como consecuencia el abandono del realismo en metafisica. La expresión hace referencia a la argumentación desarrollada por Kant a lo largo de varias páginas en el Prólogo a la Segunda Edición de la Crítica de la razón

${ }^{2}$ Cf. SERGIO RABADE, La noción de experiencia en el empirismo ingles. Hume, en Diálogos (Rio Piedra. Puerto Rico), 24 (1973), pp. 33-51. 
pura (B XIII-XVI). La inversión que supondría el giro copernicano es enunciada por Kant en los siguientes términos: «Se ha supuesto hasta ahora que todo nuestro conocer debe regirse por los objetos... Intentemos, pues, por una vez, si no adelantaremos más en las tareas de la metafísica suponiendo que los objetos deben conformarse a nuestro conocimiento, cosa que concuerda ya mejor con la deseada posibilidad de un conocimiento a priori de dichos objetos, un conocimiento que pretende establecer algo sobre éstos antes de que nos sean dados. Ocurre aquí como con los primeros pensamientos de Copérnico. Este, viendo que no conseguía explicar los movimientos celestes si aceptaba que todo el ejército de estrellas giraba alrededor del espectador, probó si no obtendría mejores resultados haciendo girar al espectador y dejando las estrellas en reposo" ( $C_{r p}, \mathrm{~B}$ XVI)

Recordemos que la reflexión de Kant tiene como guía en estas páginas la cuestión acerca de la cientificidad de la metafísica: «¿A qué se debe entonces que la metafísica no haya encontrado todavia el camino seguro de la ciencia?» (Crpp, B XV). El giro copernicano se produce como consecuencia de la elaborada y compleja respuesta de Kant a esta cuestión, e involucra los aspectos fundamentales de su pensamiento: la revisión de la metafísica escolar, la crítica entendida como el autoexamen de la razón finita, el idealismo trascendental entendido como la justificación de la posibilidad del conocimiento a priori y, por último, el aspecto sobre el que centraremos nuestra atención preferentemente: la concepción de la experiencia. En lo que sigue realizaremos una aproximación a este complejo panorama recurriendo al punto de vista histórico-evolutivo ${ }^{3}$, es decir, procurando obtener recursos para la interpretación del pensamiento de Kant a partir del estudio de los diferentes estadios evolutivos que pueden ser identificados en los textos.

3 Esta expresión quiere ser la traducción al castellano del término alemán Entwicklungsgeschichte, con el que designamos uno de los submétodos de investigación historiográfica característicos de la historiografia alemana contemporánea. Junto con la Problemgeschichte (historia del problema), la Begriffyeschichte (historia del concepto), la Quellengeschichte (historia de las fuentes), la Universitätogeschichte (historia de la universidad y otras instituciones) y la Wirkungsgeschichte (historia efectual), forma parte del utillaje habitual del investigador a la hora de reconstruir e interpretar el texto. Debemos a Dilthey el planteamiento del punto de vista histórico-evolutivo como criterio para la compilación y la edición de la obra de Kant en el gran proyecto de la Akademie-Ausgabe, cf. su Prólogo en el primer volumen de esta edición, Kant's Gesammelte Schriften, Walter de Gruyter, Berlín, 1900 y ss., vol. 1, pp. V-XV. (A partir de este momento citaremos la Akademie-Ausgabe con la abreviatura AA). 
La indagación desde el punto de vista histórico-evolutivo permite vislumbrar dos momentos estructurales en el giro copernicano, que corresponden a sendos momentos evolutivos claramente diferenciados. Hablaremos así del 'giro de la sensibilidad', que se verifica en la Dissertatio de 1770, y del 'giro del entendimiento', cuyo planteamiento aparece en la Carta a Markus Herz del 21 de Febrero de 1772 y que se consuma en los pasajes de la deducción trascendental de las categorías de la Crítica de la razón pura, en los que adquiere carta de naturaleza la ontología de la experiencia kantiana.

El punto de vista histórico-evolutivo arroja luz sobre los motivos intelectuales de Kant y al mismo tiempo constituye una guía para la comprensión de ciertas ambiguiedades que planean sobre el complejo texto de la deducción trascendental.

3. Los momentos del giro copernicano: el giro de la sensibilidad (1770) y el giro del entendimiento (1772)

El giro que Kant imprime a la indagación epistemológica se articula en un primer estadio sobre el punto de vista de la mediación de la intuición: «...es el objeto (en cuanto objeto de los sentidos) el que se rige por la naturaleza de nuestra facultad de intuición» (Crp, B XVII). Esta afirmación, extraída del pasaje en que Kant analiza y comenta el giro copernicano, en el prólogo a la segunda edición de la Crítica de la razón pura, reproduce, tomada aisladamente, el status quaestionis de la Dissertatio de 1770, obra clave en la que Kant asienta algunos presupuestos básicos del idealismo trascendental. La teoría de la idealidad de espacio y tiempo como formas de la sensibilidad aparece formulada en este escrito. Y también aquí se explicitan las consecuencias fundamentales de esta posición: el fenomenismo del mundo sensible en contraste con el realismo de un mundo comprendido directamente por un intelecto que no se atuviese a las condiciones de la sensibilidad. Kant ofrece en este contexto un primer análisis de la experiencia, que, si bien resulta rudimentario en relación con desarrollos ulteriores de su pensamiento, contiene ya algunas notas decisivas de cara a la superación de los precedentes tanto empiristas como racionalistas.

La experiencia se considera ya en esta etapa incipiente del idealismo trascendental, no meramente como lo dado, sino como el producto resultante de aplicar la actividad del intelecto al material que ofrecen los sentidos a través de una serie de operaciones (comparación, subordinación, examen según el princi- 
pio de no-contradicción) que Kant engloba bajo la expresión 'uso lógico' del intelecto: «En lo sensible y en el fenómeno, lo que antecede al uso lógico, se llama apariencia, y se llama experiencia el conocimiento reflejo originado a partir de múltiples apariencias comparadas por el intelecto. No se da, por consiguiente, camino de la apariencia a la experiencia sino por medio de la reflexión, según el uso lógico del intelecto" (Dis., \$ $\$)^{4}$. El contraste con la 'apariencia' determina esta primera acepción kantiana de 'experiencia'.

Norbert Hinske ha reflexionado en un artículo reciente ${ }^{5}$ acerca de la proximidad y la distancia del punto de vista de Kant en la Dissertatio con respecto a Meier, autor del compendio de lógica que Kant utilizó durante toda su vida académica. Es característico de la acepción de Meier, en línea con lo que veníamos observando acerca del punto de vista empirista, el considerar a la experiencia como un punto de partida en el proceso gnoseológico. Hay que hacer referencia en primer lugar a la clasificación de los conceptos, que Meier enfoca desde un punto de vista genético, es decir, considerando su origen: "Tenemos solamente tres caminos para acceder a conceptos: la experiencia, la abstracción y el enlace arbitrarion ${ }^{6}$. Eliminado en Meier el patrimonio racionalista de las ideas innatas, nos encontramos aquí con el doblete 'concepto de experiencia'-'concepto abstracto', al que cabe añadir lo que parece la réplica de las cartesianas ideae a me ipso factae'. La definición de 'experiencia' que Meier proporciona en su manual de lógica constituye una de las muchas expresiones de la sutil fusión entre el punto de vista del empirismo y del racionalismo que se ha producido en el contexto de la filosofia escolar ${ }^{8}$. Meier recurre todavía al vocabulario característico del análisis de la conciencia y del criterio de verdad cartesianos y, en craso contraste con el

${ }^{4}$ Cito la Dissertatio (= Dis.) siguiendo la versión castellana de José Gómez Caffarena, Kant, Principios formales del mundo sensible y del inteligible (Disertación de 1770), CSIC, Madrid, 1996, aquí p. 12.

${ }^{5}$ NORBERT HINSKE: Wandlungen in Kants Verstandnis von Erfahrung, en Lessico Intellettuale Europeo, Experientia, X Colloquio Internazionale, Roma, 4-6 gennaio 2001, Leo Olschki Editore, Roma, 2002, pp. 425-434.

${ }^{6}$ MeIER, Auszug aus der Vernunftlehre, reimpreso en AA, vol. XVI, Berlín - Leipzig, 1924 (1914), p. 541.

7 Cf. HINSKE, op. cit., p. 429.

${ }^{8}$ GEORGE FRIEDRICH MEIER (1718-1777) se ubica en la rica tradición de la filosofía escolar de la ilustración alemana: la saga de los filosofos-profesores inaugurada por Christian Thomasius y seguida por Christian Wolff y por el propio Kant, que harán florecer la ilustración en el seno de la institución universitaria. Con respecto a la penetración del empirismo en este contexto, es preciso tener en cuenta la todavía poco estudiada recepción de la obra de Locke. 
punto de vista racionalista, admite un tipo de claridad propio de las representaciones provenientes de los sentidos: "La experiencia (experientia) consiste [...] en aquel tipo de conocimiento, que es claro a través de la sensación"?. La experiencia equivale al conocimiento sensible. Sobre los conceptos de experiencia se forjan, mediante elaboración intelectual, los conceptos abstractos. Las lecciones de lógica, que recogen las explicaciones kantianas del manual de Meier, ponen de manifiesto las diferencias entre el punto de vista kantiano y el de su predecesor. Todos los ejemplares de lecciones conservados son posteriores a 1770 . El concepto de experiencia de la Dissertatio ha dejado aquí su huella. El doblete 'concepto de experiencia'-'concepto abstracto' se fusiona en una única acepción: los conceptos de experiencia son, para Kant, precisamente los conceptos abstractos, es decir, los productos del uso lógico del intelecto. Así, en la Lógica Blomberg, de datación cercana a la Dissertatio: "El origen primero de nuestro conocimiento es la experiencia, conceptos de experiencia (Erfahrungs-Begriffe), son por tanto [aquellos conceptos], que han sido dados por la experiencia y que se han convertido en generales mediante la abstracción. Por lo tanto todos los conceptos de experiencia son conceptos abstractos» ${ }^{10}$. A la acepción de experiencia como el resultado de los procesos de elaboración lógica de los conceptos es preciso sumar, en la etapa evolutiva correspondiente al status quaestionis de la Dissertatio, los procesos de racionalización característicos del conocimiento científico, equivalentes a la formulación de leyes cada vez más universales en el ámbito empírico: «Los conceptos comunes de la experiencia se llaman empíricos, y los objetos fenómenos, y las leyes de la experiencia y en general de todo conocimiento científico se llaman leyes de los fenómenos" (Dis., $₫ 5)^{11}$.

En la Dissertatio Kant ofrece tan solo brevísimas pinceladas en relación con los rudimentos de una nueva teoría de la experiencia. Sus observaciones en este contexto parecen dirigidas ante todo a hacer patente el hecho de que las leyes del mundo empírico, por muy generales y abstractas que sean, nunca pierden su condición de conocimiento sensible-empírico: «Así, pues, los conceptos empíricos no se hacen intelectuales en sentido real por su reducción a una mayor universalidad, y no exceden el modo del conocimiento sensible, sino que permanecen indefinidamente sensibles, por mucho que se eleven por abstracción ${ }^{12}$.

\footnotetext{
9 MEIER, op. cit., p. 493.

${ }^{10} \mathrm{KANT}, \mathrm{AA}$, vol. XXIV, p. 254.

$11 \mathrm{KANT}$, Dis., cit., p. 12.

12 Ibid.
} 
El conocimiento sensible responde a las condiciones que fija la subjetividad humana en su acceso cognoscitivo al mundo. En la Dissertatio: "Por consiguiente, todo lo que hay de sensible en el conocimiento depende de la índole especial del sujeto" ". ${ }^{13}$. Estas condiciones son, a la vez, expresión de la finitud del ser humano, expuesto a lo dado, a aquello que se le presenta pero que él mismo no ha generado. En el mismo pasaje: "La sensibilidad es la receptividad de un sujeto, por la que es posible que el estado representativo del mismo sea afectado de determinada manera por la presencia de algún objeton ${ }^{14}$.

La cuestión que se plantea a raíz de la nueva teoría de la sensibilidad de la Dissertatio puede formularse en los siguientes términos ¿sería posible, para una inteligencia limitada por la sensibilidad, un acceso al orden de lo real que no se atenga a las condiciones del conocimiento sensible? En este escrito Kant parece estar tomando como punto de partida precisamente esta hipótesis: «La inteligencia (racionalidad) es la facultad de un sujeto, por la cual es capaz de representarse lo que por razón de su condición no puede penetrar en sus sentidos" ${ }^{15}$.

El Kant de la Dissertatio, al postular la autonomía del conocimiento sensible, establece también la imposibilidad de la reducción del conocimiento sensible al inteligible. No existe una mera diferencia de grado entre conocimiento sensible e inteligible, tal como estipulaba el racionalismo. El conocimiento de origen sensible es y permanece sensible por mucho que sea sometido a procesos de elaboración intelectual. La experiencia, identificada con el ámbito del conocimiento sensible, queda al mismo tiempo estrictamente contrapuesta al ámbito de la realidad en sí. Pero a diferencia del empirismo, que, apoyándose en el presupuesto del conocimiento sensible, había renunciado a la investigación de la realidad en sí, Kant se compromete en este escrito con un objetivo tal, con el fin, como veremos, de salvaguardar por el momento el estatus y las aspiraciones de la metafísica. Así pues, en $\mathbf{1 7 7 0}$ la teoría kantiana de la experiencia aborda el problema del conocimiento sensible desde la perspectiva del uso lógico del intelecto, por otra parte Kant se propone una teoría del conocimiento no sensible. Estos elementos son suficientes para reconocer que el pensamiento de Kant ha adoptado ya en este momento la vía intermedia entre el racionalismo y el empi-

${ }^{13}$ Ibid., $\$ 4$, p. 10 .

${ }^{14}$ Ibid., $\$ 3$, p. 10.

15 Ibíd. 
rismo a la que tantas veces se ha recurrido como argumento para la presentación de su pensamiento. El punto de vista del empirismo es superado en la Dissertatio al establecer una doble mediación en el conocimiento: la mediación de las formas de la sensibilidad que convierte el dato empírico en 'apariencia' y la mediación intelectual que convierte la 'apariencia' en 'experiencia'. El punto de vista del racionalismo es superado también toda vez que se establece la autonomía del conocimiento sensible y su irreductibilidad a conocimiento inteligible: «Por lo dicho se ve que se declara mal lo sensible [diciendo que es] lo conocido confusamente y lo intelectual [diciendo] que es aquello cuyo conocimiento es distinto... Temo que el clarísimo Wolff con aquella diferencia entre sensible e intelectual, que para él es puramente lógica, anule por completo la excelente doctrina de la antigüedad acerca de la constitución de los fenómenos y noúmenos, con mucho dańo de la filosofía, y así aparte los ánimos de su indagación para llevarlos a las que son muchas veces puras minucias lógicas»" ${ }^{16}$. La apuesta por la indagación de los fundamentos del conocimiento intelectual puro, completamente independiente del conocimiento sensible, ha de ser vista también como una toma de posición con respecto al punto de vista del racionalismo wolffiano. Recordemos la definición de metafísica en el manual de Baumgarten: "Metaphysica es scientia primorum in humana cognitione principiorum" ${ }^{17}$. La metafísica es la ciencia de los primeros principios del conocimiento humano, un proyecto de metafísica en línea con la orientación gnoseológica que Descartes había impreso a la filosofía primera ${ }^{18}$. El racionalismo wolffiano, apoyado en una concepción de la verdad que estipula la continuidad o mera diferencia de grado entre el conocimiento sensible y el inteligible, se había comprometido con la construcción de un gran sistema intentando buscar las conexiones racionales entre los diversos conceptos para establecer así la concatenación universal de los saberes ${ }^{19}$. Este ideal de con-

${ }^{16}$ Ibíd., $\$ 7$, p. 13

${ }^{17}$ Alexander GotTlieb Baumgarten, Metaphysica, $\$ 1$, Halle 1739 , reimpresa en AA, vol. XVII, aquí p. 23)

${ }^{18}$ Cf. RENE Descartes, Principios de filosofla, Gredos, Madrid, 1989, Prefacio, p. 22: «... la verdadera filosoffa, cuya primera parte es la metafísica, que contiene los principios del conocimiento, entre los cuales está la explicación de los principales atributos de Dios, la de la inmaterialidad de nuestras almas, y la de todas las nociones claras y simples que hay en nosotros... Así toda la filosofia es como un árbol, cuyas raices son la metafísica, el tronco la física, y las ramas que salen de este tronco son todas las demás ciencias..."

${ }^{19}$ Cf. Chrustian WolfF, Pensamientos racionales acerca de Dios, el mundo y el alma del hombre, asi como sobre todas las cosas en general (Metafisica alemana), Akal, Madrid, 2000, p. 51-52: "Es así que he llevado distinción a los primeros fundamentos del conocimiento, de los cuales sólo se tenían o conceptos confusos o incluso totalmente oscuros, de manera que se los pueda utilizar 
tinuidad y concatenación racional es puesto en entredicho con la estricta separación entre conocimiento sensible y conocimiento inteligible de la Dissertatio. Queda en suspenso, por tanto, la posibilidad de un paso sin fisuras que nos lleve del conocimiento científico al metafísico y/o viceversa, tal como se contemplaba en el proyecto acariciado por el racionalismo. De este punto de vista se derivan consecuencias de enorme importancia para lo que será la reconducción kantiana del problema de la metafísica. Está en juego el papel de la metafísica como "reina de todas las ciencias" ( $C r p$, A VIII). La investigación de la realidad en sí deberá proporcionar un tipo de conocimiento, que, al situarse al margen del conocimiento de tipo sensible o empírico, solamente puede tener su origen en las mismas capacidades intelectuales humanas, a las que Kant, en la medida en que las considera capaces de generar por sí mismas conocimiento, les atribuye un uso que denomina 'real': "Por lo que toca a lo intelectual en sentido estricto, acerca de lo cual el uso del intelecto es real, ... tales conceptos, tanto de los objetos como de sus relaciones, se dan por la misma naturaleza del intelecto, y no son abstraídos a partir de ejercicio alguno de los sentidos, ni contienen forma ninguna del conocimiento sensible en cuanto tal ${ }^{20}$. La metafísica es ahora un saber que emana exclusivamente del intelecto puro: "La filosofia que contiene los primeros principios del uso del intelecto puro es la metafísica $^{21}$. La consecuencia inmediata de este punto de vista es la estricta separación entre ciencia y metafísica, entre conocimiento empírico y conocimiento no empírico. Nos hallamos por el momento en las antípodas del proyecto que hemos denominado aquí la kantiana 'ontología de la experiencia'.

La valoración acerca de las relaciones entre metafísica y experiencia en la Dissertatio se torna un poco más compleja si se tiene en cuenta una ulterior elaboración teórica presente en este escrito, que aparece decididamente como un adelanto de lo que será la posterior filosofia de Kant. Se trata de la teoría de la

en silogismos, donde, antes de esto, por falta de los mismos, se tenían que enjuiciar las cuestiones de modo imperfecto mediante fundamentos externos. Y ello no es poco importante, ya que, en último extremo, todas las ciencias, se las acote como quiera, van a desembocar a estos fundamentos." Wolff expone el proyecto completo del sistema de las ciencias y la concatenación entre sus partes en el capítulo III del Discursus praeliminaris de philosophia in genere/Einleitende Abhandlung über Philosophie im Allgemeinen, Frommann-Holzboog, Stuttgart-Bad Cansstatt, 1996, ritulado De partibus philosophiae, pp. 66-125.

${ }^{20} \mathrm{KaNT}, D$ is, cit., $\$ 6$, p. 12 .

21 Ibld., $\$ 8$, p. 13. 
'adquisición originaria'22 de los conceptos puros, según la cuál, éstos, si bien se originan en el intelecto, deben su génesis a la 'experiencia': «Así pues, no dándose en la metafísica principios empíricos, los conceptos en ella presentes no se han de buscar en los sentidos, sino en la misma naturaleza del intelecto puro, no como conceptos innatos, sino como abstraídos de las leyes connaturales de la mente (habida cuenta de su ejercicio con ocasión de la experiencia) y son por lo mismo adquiridos ${ }^{23}$. En primer lugar parece que lo que hayamos de entender aquí por experiencia no se corresponde con la noción de experiencia como el resultado del uso lógico del intelecto, sino más bien con el momento pre-lógico o pre-intelectual del conocimiento. Algunas Reflexiones proporcionan indicios de que esto es así: «...pero las nociones racionales surgen, sí, por medio de las sensaciones y pueden ser pensadas sólo en aplicación a las ideas abstraídas de ellas, pero no radican en ellas y no son abstraídas de ellas" ${ }^{24}$. Conforme a la teoría de la adquisición originaria, los conceptos puros, que nos proporcionarían un conocimiento 'real' acerca de las cosas, es decir, un tipo de conocimiento no mediado por las condiciones de la sensibilidad, surgirían en el intelecto con ocasión del propio conocimiento sensible.

Algunas de las ambigüedades que planean sobre el denso y conciso texto de la Dissertatio no tardarán en clarificarse. Pronto se hará visible una ulterior dimensión del giro copernicano, relativa no solamente a la mediación del conocimiento de objetos a través de las formas de la sensibilidad, sino a las condiciones interpuestas por el intelecto. Este avance decisivo en el giro, que teñirá de un nuevo barniz las nociones en torno a las que se articulaba el pensamiento de la

${ }^{22}$ La doctrina de la adquisición originaria de las representaciones puras (conceptos puros e intuiciones puras) constituye una pieza fundamental del idealismo trascendental kantiano que con frecuencia pasa desapercibida. Kant supera el innatismo racionalista a través de esta concepción. Esta doctrina es, al mismo tiempo, una de las expresiones más logradas de la dessustancialización del cogito cartesiano operada por Kant. Las facultades cognoscitivas se convierten en instancias de carácter meramente operacional-formal, cuyas funciones se activan con ocasión de la experiencia, generando las respectivas formas a priori. Un estudio exhaustivo de esta doctrina desde el punto de vista histórico-evolutivo se encuentra en MICHAEL OBERHAUSEN, Das neue a priori. Kants Lebre von einer 'ursprünglichen Erwerbung' apriorischer Vorstellungen, ed. FrommannHolzboog, Stuttgart-Bad Cannstatt, 1997.

${ }^{23}$ KANT, Dis., cit., $\$ 8$, p. 13.

${ }^{24}$ Refl. 3930, de la famosa fase $\mathrm{k}$, correspondiente a 1769. Contamos con una traduccion al castellano de la Reflexión incluida en la selección de textos que acompaña a la edición de la Dissertatio de Jose Gomez CAFFARENa, Dis., cit., p. 43. 
época: la noción de experiencia, la noción de objeto, la noción de conocimiento, y que elucidará definitivamente el carácter del proyecto de metafísica de la Dissertatio, se lleva a cabo en el momento en que Kant intenta proporcionar una explicación plausible y completa del fundamento de la relación entre el conocimiento y el objeto. Los diferentes aspectos de esta cuestión aparecen claramente formulados en la Carta a Marcus Herz del 21 de febrero de 1772. Esta misiva constituye un compendio de los problemas que la Dissertatio había dejado sin solución y apunta las rutas que en adelante tomará el pensamiento de Kant. Es para muchos el testimonio del 'giro gnoseológico' que conducirá la metafisica a nuevas posiciones. Existe un problema general, que afecta tanto al conocimiento sensible como al inteligible: "¿Cuál es el fundamento sobre el que reposa la relación de lo que llamamos representación al objeto? ${ }^{25}$. El conocimiento sensible implica referencia a objetos. Referencia que Kant, ya desde la Dissertatio, tematiza bajo el punto de vista de la 'receptividad' (receptivitas). Pero ¿cómo explicar el conocimiento en el que no está presente la receptividad? En los términos de la famosa carta: "¿Cómo puede mi entendimiento formar para sí totalmente a priori conceptos de cosas con los que necesariamente han de concordar las cosas?" ${ }^{26}$. Kant introduce a continuación una observación que resuelve las ambigüedades en relación con la problemática de la experiencia en la Dissertatio, aproximándose a la orientación que ha de tomar esta noción en la Crítica de la razón pura: "¿Cómo puede [mi entendimiento] proyectar principios reales sobre su posibilidad, [la posibilidad de las cosas] con los cuales habrá la experiencia de concordar fielmente, siendo así que son independientes de ella? ${ }^{27}$ El problema que formula este pasaje es el problema de la relación entre los conceptos intelectuales puros y la experiencia. En la Dissertatio Kant ofrece un primer catálogo de estos conceptos: «... son de esta clase [los conceptos de] posibilidad, existencia, necesidad, sustancia, causa, etc. ${ }^{28}$. Como anunciaba la Refl. 3930 , citada anteriormente, la mediación intelectual no corresponde ahora únicamente a las operaciones características del uso lógico del intelecto, sino que también los conceptos que se generan en el uso real han de ser pensados en relación con la experiencia de los objetos. La cuestión ahora encierra la siguiente paradoja ¡cómo explicar la correspondencia entre conceptos completamente

${ }^{25}$ KaNT, Carta a Marcus Herz en ibíd., p. 48.

${ }^{26}$ Ibid., p. 49.

${ }^{27}$ Ibid.

${ }^{28}$ KaNT, Dis., cit., $\$ 8$, p. 13. 
independientes de la experiencia y la experiencia? Lo que en la Dissertatio se denominaba 'mundo inteligible', el mundo de la realidad 'en sf', y el mundo fenoménico representan ahora dos caras de un mismo problema, problema al que es preciso proporcionar una solución unitaria. Pensar las cosas mediante el elenco de conceptos característicos del uso real intelectual, y conocer los objetos a través de la experiencia sensible son procesos que no pueden aparecer totalmente desconectados. Será precisamente la conexión de los conceptos puros con el ámbito del conocimiento fenoménico lo que dará pie a la transformación de la noción kantiana de experiencia. Ya en las Reflexiones del legado manuscrito de Duisburg se reconoce este nuevo punto de vista: Refl. 4679, fase $=1775$ : "Experiencia es una percepción comprendida. La comprendemos empero cuando nos la representamos bajo títulos del entendimiento ${ }^{29}$. Reuniendo en una sola frase la doctrina de la adquisición originaria de los conceptos puros y la explicación de la función de los mismos, que ha de ser pensada en relación con la experiencia, la Refl. 4172: "Los conceptos dados mediante la razón antes de toda experiencia, aunque abstraídos con ocasión de la experiencia a partir de las leyes de la razón, p. ej. el de razón (Grund), son formas impresas (eingedrükte) en el entendimiento ordenador (dem ordnenden Verstande) para uso de las experiencias" ${ }^{30}$. Por otra parte las Reflexiones permiten paralelamente vislumbrar como se va perfilando la noción kantiana de 'mundo inteligible', noción que ya no se corresponde con un proyecto de metafísica entendida como conocimiento teorético de las cosas tal como son en sí mismas, sino que queda restringida al ámbito práctico de la moralidad y la libertad. Desde el punto de vista del conocimiento, el mundo es únicamente mundo sensible, mientras que el mundo inteligible se corresponde a la realidad metafísica de la libertad. Refl. 4349 (varias fases posibles, las más probables $\lambda=$ aprox. 1770-1771, $\rho=$ aprox. 1775-1777): "A nosotros no puede sernos dado otro mundo que no sea el sensible; por tanto, todo mundus physicus, (materialiter) es sensibilis; sólo el mundus moralis (formaliter) es intelligibilis. Por eso, porque la libertad es lo único que es dado a priori y consiste en este dar a priori (und in diesem Geben a priori besteht); la regla de la libertad a priori en un mundo en general constituye la formam mundi intelligibilis ... El mundus intelligibilis como un objeto de la intuición es una mera idea (indeterminada); pero como un objeto de la relación práctica de nuestra inteligencia con las inteligencias del mundo en general y con Dios como la esencia

${ }^{29}$ KANT, AA, vol. XVII, p. 664.

30 Ibid., p. 443. 
originaria práctica (dem praktischen Urwesen) de las mismas, es un concepto verdadero y una idea determinada: civitas dei ${ }^{31}$. También la Refl. 4254 (la fase más probable $K=$ aprox. finales de 1769-otono de 1770): «El mundo inteligible es aquél cuyo concepto es válido para todo mundo, por consiguiente no contiene leyes físicas, sino leyes objetivas y morales... El concepto intelectual del mundo es por tanto el concepto de perfección. El mundo inteligible es el moral y las leyes del mismo tienen validez para todo mundo como leyes objetivas de la perfección ... Mundus intelligibilis es el mundo de los seres racionales, considerado conforme a leyes objetivas de la libertad $»^{32}$.

Como consecuencia de lo que hemos denominado aquí 'giro del entendimiento', es decir, del segundo momento del giro copernicano, el problema gnoseológico queda circunscrito al ámbito de la razón limitada por la sensibilidad, es decir, estrictamente al ámbito de la razón finita.

\section{Experiencia y objetividad: la deducción trascendental de las categorías}

Las páginas introductorias de la deducción trascendental de las categorías, inalteradas en la segunda edición de la Crítica de la razón pura, contienen ciertos pasajes muy significativos con respecto a la evolución intelectual de Kant. Vamos a ver que las expresiones de Kant en estos pasajes no se corresponden a la solución definitiva del problema, que apunta a la articulación entre los conceptos puros y la experiencia, sino que reproducen el status quaestionis característico de las etapas evolutivas anteriores, destinadas a ser superadas precisamente en estas páginas de la Critica de la razón pura.

El primero de los pasajes que aducimos reproduce la cuestión que planteaba la Carta a Marcus Herz de 1772: "Con los conceptos puros del entendimiento empieza, en cambio, la necesidad ineludible de buscar la deducción trascendental... En efecto, al tratar de objetos mediante predicados del pensamiento puro a priori, y no mediante predicados de la intuición y la sensibilidad, se refieren a objetos en sentido universal, prescindiendo de todas las condiciones de la sensi-

${ }^{31}$ Ibid., p. 516.

32 Ibíd., p. 483-484. 
bilidad. Como tales conceptos no se basan en experiencia, tampoco pueden aducir objeto alguno sobre el que fundamentar su síntesis previa a toda experiencia.» (Crp, A 88/B 120-121).

El siguiente fragmento, que aparece en páginas sucesivas, se retrotrae incluso más atrás, puesto que reproduce el status quaestionis alcanzado en la Dissertatio, que proporcionaba una primera respuesta al problema del conocimiento sensible, pero que dejaba pendiente el problema del tipo de conocimiento que se puede obtener a través de los conceptos puros o categorías: «Las categorías del entendimiento no nos representan, en cambio, las condiciones bajo las cuales se nos dan objetos en la intuición. Por consiguiente, se nos pueden manifestar objetos sin que tengan que referirse forzosamente a funciones del entendimiento y sin que, por tanto, el entendimiento contenga a priori las condiciones de los mismos. Tal es la razón de que aparezca en este punto una dificultad que no hemos hallado en el terreno de la sensibilidad, a saber, cómo pueden tener validez objetiva las condiciones subjetivas del pensar, es decir, cómo pueden éstas proporcionar las condiciones de posibilidad de todo conocimiento de los objetos, ya que desde luego, pueden darse fenómenos en la intuición con independencia de las funciones del entendimiento" (Crp, A 90/B 122).

Al estudioso de la filosofia de Kant no dejarán de sorprenderle ciertas afirmaciones contenidas en estos pasajes. En la deducción trascendental se pondrá de manifiesto que los conceptos puros, si es que encierran algún tipo de conocimiento, no podrán situarse completamente al margen de las condiciones de la sensibilidad. Por otra parte, será refutado el postulado de una hipotética independencia de los objetos con respecto a las condiciones del pensar. Es esta una conclusión a la que Kant se aproxima con toda la cautela de quien presenta un descubrimiento que encierra gran parte del potencial innovador de su filosofía: «... el que los objetos de la intuición sensible hayan de conformarse, además, a las condiciones que el entendimiento exige para la unidad sintética del pensar es una conclusión que no es tan fácil de ver” ( $C r p$, A 90/B 124). El grado de dificultad al que Kant se enfrentaba en estas páginas puede justificar su vacilante redacción, que, como ha señalado Paton, más parece un patch-work que un argumento correctamente hilvanado.

Con respecto a lo que nos interesa aquí: la modificación de la noción de experiencia en Kant y la formulación de las claves de lo que hemos denominado 'ontología de la experiencia', baste señalar que es justamente el empleo de la 
noción de 'objeto' el que está destinado a sufrir importantes modificaciones, al hilo de las cuales veremos transformarse la propia noción de experiencia. El problema general que Kant intenta resolver en la deducción trascendental: la cuestión de la validez objetiva de las categorías, se enfoca de dos maneras diferentes, la argumentación presenta, según su propia expresión, dos vertientes (zwei Seiten), la deducción objetiva (con el punto de partida en el objeto) y la deducción subjetiva (con el punto de partida en el sujeto). En palabras de Kant: «La primera se refiere a los objetos del entendimiento puro... La segunda trata de considerar el entendimiento mismo" (Crp, A XVI). La deducción objetiva vincula las categorías a la experiencia precisamente a través de la noción de objeto. La novedad de estas páginas consiste en considerar ahora que la experiencia tiene como requisito imprescindible, además de lo dado a la intuición sensible, el concepto de un objeto: «Pero resulta que toda experiencia contiene, además de la intuición sensible mediante la cual algo está dado, el concepto de un objeto dado o manifestado en la intuición. Consiguientemente, habrá conceptos de objetos que, como condiciones a priori, sirvan de base a todo conocimiento experimental. La validez objetiva de las categorías como conceptos a priori residirá, pues, en el hecho de que sólo gracias a ellas sea posible la experiencia (por lo que hace a la forma del pensar)" (Crp, A 93/B 126). Kant tiende a través de la noción de objeto el puente que necesitaba entre las condiciones de la sensibilidad y las condiciones del entendimiento. También en este punto se produce un avance decisivo con respecto al empirismo. Xavier Zubiri ha sintetizado en una elocuente expresión el punto de vista de Kant en el momento definitivo del giro copernicano: «el ser objeto no está dado" ${ }^{33}$. Para que algo sea objeto tiene que ser pensado, y a la vez no podemos hablar de experiencia más que como experiencia de objetos. La experiencia incluirá por tanto las condiciones del pensar. Clarificado por Zubiri: «Experiencia no significa para Kant simplemente la experiencia sensible o, como se dice vulgarmente, las sensaciones o las percepciones sensibles; experiencia significa el sistema de objetos sensibles" ${ }^{34}$.

Kant se verá obligado a desglosar las componentes que integran la posibilidad del pensamiento de objetos en el ámbito de la experiencia. Dicho de otro modo: merced a la mediación intelectual, Kant conseguirá convertir a la 'expe-

${ }^{33}$ CF. XAVIER ZUBIR, Los problemas fundamentales de la metafisica occidental, ed. Alianza Universidad, Madrid, 1995, p. 192.

34 Ibid., p. 215. 
riencia' en correlato de 'objetividad' y ello en la medida en que el pensamiento puro de objetos, que representan los conceptos puros o categorías, se contempla como condición misma de la experiencia. Si el primer momento del giro copernicano consistia en convertir los objetos de la sensibilidad en fenómenos, el segundo momento consiste en convertir en problema la misma noción de objeto, tornándola comprensible desde las condiciones que interpone el intelecto humano. La noción de experiencia se consolida como equivalente al conocimiento de objetos a través de conceptos. La experiencia requiere la mediación del concepto y por tanto, deberá regirse por ciertas reglas que tienen su fundamento en el entendimiento mismo como facultad de los conceptos: «... la misma experiencia constituye un tipo de conocimiento que requiere entendimiento y éste posee unas reglas que yo debo suponer en mí ya antes de que los objetos me sean dados, es decir, reglas a priori. Estas reglas se expresan en conceptos a prio$r i$ a los que, por tanto, se conforman necesariamente todos los objetos de la experiencia y con los que deben concordar» ( $C_{r p}$, B XVIII).

El giro copernicano completo (el giro de la sensibilidad y el giro del entendimiento) expresa entonces la doble dimensión de la finitud humana. De nuevo en palabras de Zubiri: "La razón es finita porque conoce de otra manera, que consiste en conocer algo que es recibido en una intuición sensible y, además, porque esto que es recibido tiene que ser hecho objeto de pensamienton ${ }^{35}$.

Kant desarrolla ampliamente su nueva teoría del objeto en la primera versión (Crp, A 104/B 110) de la deducción, y la presenta en apenas una frase en la segunda edición: "Objeto es aquello en cuyo concepto se halla unificado lo diverso de una intuición dada" ( $C_{r}$, B 137). Lo mismo que la experiencia en general aparece como resultado, el objeto ha de considerarse también como un producto. La investigación genética que Kant se propone acerca del origen del conocimiento, resulta ser una investigación en la que se desvela el procedimiento de génesis del objeto del conocimiento. Este procedimiento de génesis equivale a las diferentes operaciones sintéticas que realiza el entendimiento, operaciones unificadoras que culminan en la condición suprema de unidad, es decir, en la unidad de la autoconciencia: «La unidad sintética de la conciencia es, pues, una condición objetiva de todo conocimienton ( $C_{r p}$, B 138).

\footnotetext{
${ }^{35}$ Ibíd., p. 191.
} 
Es precisamente la nueva teoría del objeto lo que permite considerar estas páginas de la Crítica de la razón pura como una ontología de nuevo cuño, que se desarrolla íntegramente a partir de las condiciones de la subjetividad humana. Existen objetos sólo en tanto que existe un 'yo' que piensa, es decir, que reduce a unidad lo dado en la intuición. Esta unidad tiene en Kant una doble dimensión: unidad en el concepto y unidad de los conceptos en una y la misma conciencia. La apercepción trascendental (Ich denke) debe poder acompañar a todas mis representaciones, a todas, es decir, tanto a las intelectuales o conceptuales como a las sensibles: «La representación que puede darse con anterioridad a todo pensar recibe el nombre de intuicion. Toda diversidad de la intuición guarda, pues, una necesaria relación con el Yo pienso en el mismo sujeto en que se halla tal diversidad." (Crp, B 132). A través de la mediación intelectual las intuiciones dejan de ser 'ciegas' y el conocimiento sensible se convierte propiamente en 'experiencia'.

Un elemento fundamental de la trama de la deducción trascendental consiste, pues, en haber pensado la experiencia a partir de la noción de objeto. Pero los resultados relevantes de estas páginas nucleares de la Crítica de la razón pura van más allá. Se trata de demostrar no únicamente que los conceptos puros tienen validez en relación con la experiencia sino que sólo tienen validez en relación con la experiencia. La tarea consiste en poner de manifiesto la copertenencia y la indisolubilidad del elemento sensible y del intelectual en el conocimiento. En estos pasajes se constituye la razón finita kantiana. Kant asume aquí con toda radicalidad las consecuencias que se derivan de la mediación del conocimiento a través de las condiciones de la sensibilidad. La verdadera fuerza probatoria de la deducción en este sentido deriva de que no cabe pensar otro modo de 'darse' objetos más que el modo sensible-empírico. "Toda intuición posible para nosotros es sensible (cf. estética). Consiguientemente, al pensar, mediante un concepto puro del entendimiento, un objeto en general sólo podemos convertirlo en conocimiento en la medidà en que refiramos este conocimiento a objetos de los sentidos» (Crp, B 147). En el siguiente párrafo, que recoge los resultados de la deducción trascendental, queda claro que, debido precisamente a que "todas nuestras intuiciones son sensibles" el pensamiento de objetos no puede ser más que pensamiento de objetos en el ámbito de la experiencia: «No podemos pensar un objeto sino mediante categorías ni podemos conocer ningún objeto pensado sino a través de intuiciones que correspondan a esos conceptos. Igualmente, todas nuestras intuiciones son sensibles y este conocimiento, en la medida en que su objeto es dado, es empírico. Ahora bien, el conocimiento empírico es la experiencia. No podemos, pues, tener conocimiento a priori sino de objetos de experiencia posiblen (Crp, B 165). 
Fijar las condiciones de la experiencia supone fijar las condiciones de la posibilidad de un mundo de objetos independiente de las percepciones subjetivas. Así, en la analítica de los principios: "... las condiciones de posibilidad de la experiencia en general constituyen, a la vez, las condiciones de posibilidad de los objetos de la experiencian (Crp, A 158/B 197). En los Prolegómenos Kant sustituye la compleja argumentación de la deducción trascendental por la distinción entre 'juicios de percepción' y 'juicios de experiencia'36. Los juicios de experiencia apuntan a un ámbito de objetividad que se constituye a través de la operación de juzgar, a la que, tal como ha quedado estipulado en la deducción metafísica, es inherente la unidad sintética que corresponde a las categorías ${ }^{37}$.

Peter Strawson, en su famoso estudio sobre la Crítica de la razon pura ha interpretado la analítica trascendental y, en concreto, los pasajes correspondientes a la deducción trascendental, como una analítica de la experiencia: «He tratado la Deducción como una argumentación que procede por medio del análisis del concepto de experiencia en general para llegar a la conclusión de que una cierta objetividad y una cierta unidad son condiciones necesarias de posibilidad de la experiencian" ${ }^{38}$. La clave de bóveda de la deducción trascendental se recogería en el siguiente enunciado: «La deducción trascendental de todos los conceptos a priori tiene, pues, un principio por el que debe regirse toda la investigación y que consiste en que tales conceptos han de ser reconocidos como condiciones a priori de la posibilidad de la experiencian (Crp, A. 94/B 127).

Desde el punto de vista de la deducción objetiva (del objeto al pensamiento), la noción de objeto se convertía en requisito de la experiencia, desde el punto de vista de la deducción subjetiva (del pensamiento al objeto), los conceptos puros miran a la experiencia y aparecen como condición de posibilidad de la misma. Son, en última instancia, funciones de unidad que hacen posible la experiencia como un todo cohesionado: «Hay sólo una experiencia en la que todas las percepciones se representan como conjuntos completos y conformes a leyes, al igual que sólo hay un espacio y un tiempo en los que se dan todas las formas del fenómeno y toda relación del ser o del no-sern ( $C_{r p}$, A 110). La transformación con respecto 145.

${ }^{36}$ Cf. KANT, Prolegómenos a toda metafisica futura, Istmo, Madrid, 1999, $\$ 18-22$, pp. 125 -

${ }^{37}$ Cf. KaNT, Crp, A 79/B 105.

${ }^{38}$ Peter STRAwSON, Los límites del sentido, Revista de Occidente, Madrid, 1975, pp. 27-28. 
al punto de vista de 1770 se recoge explícitamente en el siguiente pasaje, al hilo de la redefinición del entendimiento como 'facultad de las reglas': «El entendimiento no es, pues, una mera facultad destinada a establecer leyes confrontando fenómenos, sino que él mismo es la legislación de la naturaleza" (Crp, A 126).

Uno de los presupuestos de la superación del punto de vista del racionalismo moderno es el abandono del innatismo, hipótesis ampliamente discutida en el Libro I de los leibnizianos Nuevos Ensayos sobre el entendimiento humano. Locke toma como punto de partida el modelo de mente como tabula rasa. Hume, en su investigación de la naturaleza humana, acaba identificando el 'yo' con el conjunto de sus percepciones. El empirismo da pábulo al subjetivismo moderno en su versión psicologista, en contraste con la versión del sujeto cartesiano que toma como modelo la racionalidad matemática y con el sujeto kantiano, que recupera el punto de vista de las operaciones lógicas (concepto, juicio, raciocinio) en su análisis trascendental de la subjetividad. Kant entiende la mente como un conjunto de principios activos, tanto en el orden de la sensibilidad como en el del entendimiento y la razón. La experiencia aparece como resultado de dichos principios activos. El aspecto sustantivo de dicha actividad se puede resumir en el término 'síntesis', clave de bóveda de la teoría kantiana de la experiencia. Cuando Kant introduce la noción de síntesis en la Crítica de la razón pura, al principio de la sección que conocemos como deducción metafísica de las categorias, resalta el aspecto que engloba todas las posibles acepciones del término: síntesis es, ante todo, función de unidad que se verifica en el orden de la representación, es decir, en el orden de la conciencia: «Entiendo por síntesis, en su sentido más amplio, el acto de reunir diferentes representaciones y entender su variedad en un único conocimiento" (Crp, A 77/B 103). A través de su actividad sintética el entendimiento genera la unidad necesaria para concebir el ámbito fenoménico bajo la perspectiva de ciertas leyes universales. El modelo del uso lógico del intelecto, tal como Kant lo presentaba en la Dissertatio, ha revelado pronto su insuficiencia como marco para la justificación de la experiencia. En la Crítica de la razón pura Kant desarrolla un nuevo modelo, capaz de dar cuenta de las actividades sintéticas del intelecto. Este modelo se contiene en la ciencia denominada 'lógica trascendental'.

\section{La metafísica más allá de la experiencia}

Pero con lo expuesto hasta ahora hemos realizado únicamente una aproximación somera a la tarea que Kant impone a la razón: el autoconocimiento. En el 
Prólogo a la segunda edición de la Crítica de la razón pura Kant ha advertido acerca de la necesidad de adoptar dos puntos de vista diferentes a la hora de examinar la validez de los principios y conceptos a priori, por una parte en relación con la experiencia y por otra en relación con la posibilidad de una ampliación de los conocimientos con independencia de la experiencia. Kant habla de esta doble perspectiva como de un experimento destinado a fundamentar un nuevo método del pensamiento: «... tal experimento con conceptos y principios supuestos a priori sólo será factible si podemos adoptar dos puntos de vista diferentes: por una parte, organizándolos de forma que tales objetos puedan ser considerados como objetos de los sentidos y de la razón, como objetos relativos a la experiencia; por otra, como objetos meramente pensados, como objetos de una razón aislada y que intenta sobrepasar todos los límites de la experiencia. Si descubrimos que, adoptando este doble punto de vista, se produce el acuerdo con el principio de la razón pura y que, en cambio, surge un inevitable conflicto de la razón consigo misma cuando adoptamos un solo punto de vista, entonces es el experimento el que decide si es correcta tal distinción" (nota de Kant en Crp, B XVIII).

Esta aclaración resulta clave para comprender la evolución del proyecto de metafisica cuyos presupuestos Kant explicitaba en la Dissertatio. Los mismos conceptos que nos permiten pensar los objetos como independientes de la experiencia se aplican en definitiva al pensamiento de objetos de la experiencia. La piedra de toque para dirimir los conflictos de la metafisica: la separación entre el conocimiento sensible y el inteligible, se mantiene en esta nueva versión. Y, al igual que en la Dissertatio, esta separación es un paso previo para el desarrollo de la nueva metafísica. En otra aclaración que Kant introduce como nota al pie en su texto: "El análisis del metafisico separa el conocimiento puro a priori en dos elementos muy heterogéneos: el de las cosas en cuanto fenómenos y el de las cosas en sí mismas» (nota en Crpp, B XXI).

El conocimiento puro a priori de las cosas en cuanto fenómenos abre paso a la ontología de la experiencia, mientras que la hipótesis del conocimiento puro de las cosas tal como son en sí mismas aboca a una serie de contradicciones que se producen de modo totalmente natural en la razón, en la medida en que esta facultad se adentra en el ámbito de lo incondicionado. Para ejemplificar el valor del doble punto de vista, Kant recurre al ejemplo que probablemente le resultase más preciado: la contraposición entre causalidad y libertad. El principio de causalidad extiende su validez en el ámbito de la experiencia, en este ámbito se opone a la idea de libertad, pero considerando la distinción entre fenómeno y cosa en sí la con- 
tradicción desaparece puesto que la causalidad es un principio relativo a la organización de los fenómenos en el ámbito de la experiencia, mientras que la libertad puede decirse de un ser tomado, no como sometido a la cadena causal, sino como cosa en sí misma: «Pero si la crítica no se ha equivocado al ensefiarnos a tomar el objeto en dos sentidos, a saber, como fenómeno y como cosa en sí; si la deducción de sus conceptos del entendimiento es correcta y, por consiguiente, el principio de causalidad se aplica únicamente a las cosas en el primer sentido, es decir, en cuanto objetos de la experiencia, sin que le estén sometidas, en cambio, esas mismas cosas en el segundo sentido; si eso es así, entonces se considera la voluntad en su fenómeno (en las acciones visibles) como necesariamente conforme a las leyes naturales y, en tal sentido, como no libre, pero, por otra parte, esa misma voluntad es considerada como una cosa en sí misma y no sometida a dichas leyes, es decir, como libre, sin que se de por ello contradicción alguna" (Crp, B XXVIIXXVIII). Junto a la ontología de la experiencia se perfila, en el prólogo a la Crítica de la razón pura, el fundamento de la metafísica de la libertad.

Con respecto a la metafísica teórica, la distinción entre una ontología de la experiencia y una metafísica que no guardase ninguna vinculación con lo empírico se articula en la Crítica de la razón pura en la división entre analítica y dialéctica. La analítica aparece desde este punto de vista caracterizada como una lógica de la verdad a la que se contrapone la dialéctica como una lógica de la apariencia. La metafísica de la libertad por su parte se asienta en el sujeto entendido como razón pura práctica. El sujeto es capaz de determinarse a sí mismo a la acción en completa independencia del acontecer empírico. El yo sustancial, que Descartes contraponía a la realidad de la naturaleza concebida de acuerdo con los principios de la física matemática, se ha convertido en el yo cuya única esencia pensable sin contradicción es la libertad entendida como un principio que desplaza al ser humano fuera del orden de concatenación causal sin fisuras que establece el nuevo modelo de ciencia natural. Asi Zubiri: «Kant entiende que la libertad, es decir, que el hombre como entidad determinada inteligiblemente en forma de libertad trascendental, con causalidad libre pero en el orden inteligible, es justamente lo que constituye la persona. Para Kant, la persona es la dimensión trascendental del hombre, como cosa en sí, como cosa absoluta. De ahí que a la división cartesiana de las cosas en res cogitans y res extensa, Kant substituya la diferencia trascendental entre res y persona. Para Kant, pues, el hombre como cosa en sí es persona ${ }^{39}$.

${ }^{39}$ ZUBIRI, op. cit., p. 231. 
La ontología de la experiencia, junto con la metafísica de la libertad, puede ser vista como un nuevo tipo de dualismo, al servicio por una parte de la fundamentación del conocimiento, que queda libre de la amenaza del escepticismo, y que es capaz, por otra parte, de establecer los fundamentos de una nueva respuesta a ciertas cuestiones fundamentales relativas a la condición humana. Las tesis tradicionales de la metafísica cristiana como la inmortalidad del alma o la existencia de Dios, no pueden fundamentar la comprensión de la naturaleza humana, ni tampoco pueden proporcionar la pauta para la moralidad. La teología y sus corolarios en el ámbito de la psicología y la cosmología racionales han desaparecido del programa del conocimiento. La época de Kant, el siglo de las luces, había renunciado por motivos diversos a las indagaciones metafísicas. Kant mismo se sitúa a contracorriente. Existen varios motivos que justifican esta posición. Cabe hacer referencia en primer lugar a su talante personal, que Kant declara en aquel famoso pasaje de los Sueños de un visionario en el que se confiesa enamorado de la metafísica (Die Metaphysik, in welche ich das Schicksal habe verliebt zu sein...). En el orden de las motivaciones personales, en este caso de las puramente externas y contingentes, podemos situar el hecho de que los azares de la vida académica hubiesen llevado a Kant a tener que desempeñar una cátedra de metafísica y lógica en su universidad y a ocuparse, a lo largo de cuatro décadas, de la docencia de dichas disciplinas. Cabe citar por último su circunstancia histórica. A diferencia de Francia o Inglaterra la ilustración alemana se caracteriza por la pervivencia de las disciplinas tradicionales y por un cierto hermetismo con respecto a los avances de la ciencia moderna. Recordemos en este sentido que Federico el Grande decide en 1746 encomendar al matemático y físico francés Maupertuis la dirección de la Academia de las Ciencias de Berlín con el encargo expreso de favorecer la penetración del pensamiento de Newton en Alemania y desterrar de una vez "la barbarie leibniziano-wolffiana” ${ }^{40}$. Han transcurrido a la sazón casi sesenta años desde la publicación de los Principia de Newton. La respuesta alemana al reto de la razón ilustrada se producirá tardíamente, pero no por ello con menor intensidad. La pervivencia en las instituciones universitarias alemanas de las viejas disciplinas especulativas explica en parte que fuese precisamente en este ambiente intelectual de ilustración mitigada y retardada donde se pudiese percibir con toda claridad el conflicto entre las pretensiones de la metafísica de

40 Cf. Giorgio Tonelu, La filosofia alemana de Leibniz a Wolff, Historia de la filosofia, s. XXI Editores, vol. 7, Madrid, 1984, p. 117. 
constituirse como un saber riguroso y el Faktum de la ciencia moderna, que se ha consolidado como el nuevo modelo de este tipo de saber. Por los diversos motivos citados Kant no renuncia a la metafísica, logrando finalmente coronar el proyecto de la moidernidad al proporcionar un fundamento antropológico a la metafísica en su doble dimensión teórica y práctica. Consigue acceder hasta la raiz de aquellas cuestiones que necesariamente la razón humana formula, aunque no siempre puedan responderse.

\section{Bibliografía}

Baumgarten, Alexander Gottlieb: Metaphysica, $\$ 1$, Halle 1739, reimpresa en AA, vol. XVII, Berlín - Leipzig, 1928.

Descartes, Rene: Principios de filosofia, Gredos, Madrid, 1989.

HINSKE, NORBERT: Wandlungen in Kants Verständnis von Erfahrung, en Lessico Intellettuale Europeo, Experientia, X Colloquio Internazionale, Roma, 4-6 gennaio 2001, Leo Olschki Editore, Roma, 2002, pp. 425-434.

KanT, Immanuel: Critica de la razón pura, edición de Pedro Ribas, Alfaguara, Madrid, 1995 (1978).

- Principios formales del mundo sensible y del inteligible (Disertación de 1770), edición de José Gómez Caffarena, CSIC., Madrid, 1996.

- Kant's Gesammelte Schriften, Walter de Gruyter, Berlín, 1900 y ss., (AkademieAusgabe=AA).

- Prolegómenos a toda metafisica futura, edición de Mario Caimi, Istmo, Madrid, 1999.

MeIER, GEORGE FrIEDRICH: Auszug aus der Vernunftlebre, reimpreso en AA, vol. XVI, Berlín - Leipzig, 1924 (' $\left.{ }^{1} 1914\right)$.

OBERHAUSEN, MICHAEL: Das neue a priori. Kants Lebre von einer ursprünglichen Erwerbung' apriorischer Vorstellungen, ed. Frommann-Holzboog, Stuttgart-Bad Cannstatt, 1997.

RABADE, SERGIO: La noción de experiencia en el empirismo inglés: Hume, en Diálogos (Rio Piedra, Puerto Rico), 24 (1973), pp. 33-51.

Strawson, Peter: Los limites del sentido, Revista de Occidente, Madrid, 1975, p. 27-28.

TONELLI, GIORGIO: La filosofia alemana de Leibniz a Wolff, Historia de la filosofia, s. XXI Editores, vol. 7, Madrid, 1984, p. 117.

Wolff, Christian: Pensamientos racionales acerca de Dios, el mundo y el alma del bombre, asi como sobre todas las cosas en general (Metafisica alemana), Akal, Madrid, 2000. 
- Discursus praeliminaris de philosophia in generelEinleitende Abhandlung über Philosophie im Allgemeinen, Frommann-Holzboog, Stuttgart-Bad Cansstatt, 1996.

ZUBIRI, XAVIER: Los problemas fundamentales de la metafisica occidental, Alianza Universidad, Madrid, 1995. 